\title{
Inner skin friction of open-ended piles considering the degree of soil plugging
}

\author{
Sangseom Jeong ${ }^{\text {i) }}$ and Junyoung Ko ${ }^{\text {ii) }}$
}

i) Professor, School of Civil and Environmental Eng., Yonsei University, 50, Yonsei-ro, Seodaemun-gu, Seoul 120-749, Korea. ii) Ph.D Student, School of Civil and Environmental Eng., Yonsei University, 50, Yonsei-ro, Seodaemun-gu, Seoul 120-749, Korea.

\begin{abstract}
This paper presents an experimental study of the plugging effect on the capacity of open-ended piles installed in sandy soil. Full-scale tests, including dynamic and static axial-compression load tests, were carried out on three instrumented piles with different diameters $(508.0,711.2$ and $914.4 \mathrm{~mm})$. To measure the outer and inner shaft resistances acting on the piles, a double-walled system was utilized with instrumented strain gauges on the outside and inside walls of the pile. The results of field tests show that the inner shaft resistance was mostly mobilized at the location between the pile tip and $18-34 \%$ of the total plug length. It was found that the soil plugging in the lower portion has influence on the inner shaft resistance. In addition, a new design approach that can predict the degree of soil plugging and the inner skin friction is proposed. Based on the plugging measurements, a linear relationship between the PLR and incremental filling ratio (IFR) was proposed. Based on the results of the full-scale field pile load tests, the inner skin friction of the open-ended piles was proposed as a function of the IFR and pile diameter. The proposed method can predict the degree of soil plugging and the inner skin friction of open-ended piles and be selected as convenient option in engineering field.
\end{abstract}

Keywords: plugging effect, field load test, open-ended piles, design method, inner skin friction

\section{INTRODUCTION}

There are numerous construction projects currently under way in Korean urban and coastal areas such as harbour terminals, offshore grand bridges, and lifeline systems. Steel pipe piles are frequently used to support high-rise buildings and heavy structures for ensuring structural safety rather than service limit capacity. Steel pipe piles are also widely applied in civil engineering structures because of their high load bearing capacity, light weight and outstanding workability. Substantial foundations are required for grand bridges and high-rise buildings, and large-diameter driven piles are frequently used in these projects.

Some design methods for open-ended piles have been proposed. These methods have resulted in slightly different methodologies that can generally be classified into three groups: (1) cone-penetration test (CPT)-based design methods (Paik and Salgado, 2003; Feng and Jun, 2012; Jardine et al., 2005; Lehane et al., 2005) (2) standard penetration test (SPT)-based design methods (Lai et al., 2008), and (3) the earth pressure approach (API 2007). However, the existing design methods have some limitations, such as a heavy dependence on the correlations that are derived from the model tests and CPTs. It is not easy to design the bearing capacity of open-ended piles considering the plugging effect in common practical cases, because the degree of plugging is not well reflected in the design methods.

The overall objective of this study is to propose an open-ended pile design methodology that considers the plugging effect using the results that are derived from full-scale tests and plugging measurements. Various influencing factors of the plugging effect should be quantified and considered when designing open-ended piles. The proposed methods were validated using a field case study. The predicted values, such as the incremental filling ratio (IFR) and inner skin friction are, compared with the measured values from the pile load tests. A new design methodology has been developed to provide a basis for a preliminary design method that would be applicable to open-ended piles taking into account the plugging effect.

\section{METHODS AVAILABLE FOR PLUGGING EFFECT ON OPEN-ENDED PILES}

The plugging effect of a steel pipe pile can be divided into three conditions: unplugged, partially plugged and fully plugged. During the initial pile driving, the soil plug length in the pile is equal to the penetration depth, and the pile is in an unplugged condition. As pile penetration continues, inner shaft frictional resistance occurs between the soil plug and the inner pile. Because of this, the length of the soil plug becomes less than the penetration depth and the pile is partially plugged. 
Eventually, the pile penetrates into soil but the soil plug length does not change, thus creating a fully plugged condition. The plugging effect can be quantified by using the plug length ratio (PLR) and the incremental filling ratio (IFR). The PLR is defined as the ratio of the soil plug length to the penetration depth at the completion of pile driving. The PLR can be written as

$$
P L R=\frac{L_{i}}{D_{i}}
$$

where $L_{i}$ is the soil plug length and $D_{i}$ is the penetration depth. PLR $=0$ represents that no soil enters the pile during pile installation. The behavior of open-ended piles in this condition is similar to the behavior of closedended piles. However, PLR $=1$ represents that the soil plug length is equal to the pile penetration depth at the end of pile installation.

The IFR can be defined as the ratio of the increment of the soil plug length to the increment of pile penetration depth during pile driving. The IFR can be written as

$$
I F R=\frac{\Delta L_{i}}{\Delta D_{i}} \times 100
$$

where $\Delta L_{i}$ is the increment of soil pile length and $\Delta D_{i}$ is the increment of pile penetration depth. IFR $=0$ and 1 are the fully plugged and fully unplugged conditions, respectively, and the IFR between 0 and 100 is the partially plugged condition. The approach to predicting the IFR from the PLR would be very convenience in the design stage. Therefore, it is necessary to investigate the relationship between the PLR and IFR.

\section{IFR MEASUREMENT AND FIELD PILE LOAD TESTS}

IFR measurement and field pile load tests were conducted on full scale piles instrumented with strain gauges to investigate the relation between the plugging effect and bearing capacity. The emphasis was on analyzing the plugging effect from the soil plug in the pile so that these tests were performed on 3 test piles under various conditions, such as pile diameter and pile length.

\subsection{Site and subsurface description}

The test site was located at the Kwangyang substiuted natural gas (SNG) plant in Korea. A geotechnical investigation was performed to define the soil profile and properties at the test site as accurately as possible. The in-situ field testing was performed on two boring holes (BH-1 and $\mathrm{BH}-2$ ) with conventional sampling near the test piles and standard penetration testing (SPT). Fig. 1 presents the soil profile with borehole and schematic representation of the instrumented piles. The soil obtained by the boring and lab tests, is generally composed of a thick hydraulic fill layer with sand. The results of SPTs indicate that sand deposits at 0 to $15 \mathrm{~m}$ were in a loose state with SPT $\mathrm{N}$ values ranging from 8 to 18 . The water table was located at $2.5 \mathrm{~m}$ below the ground surface. The cohesionless soils were classified as SP or SM with round angularity according to the Unified Soil Classification System (ASTM, 2011). The properties of soils based on the site investigation are summarized in Table 1.

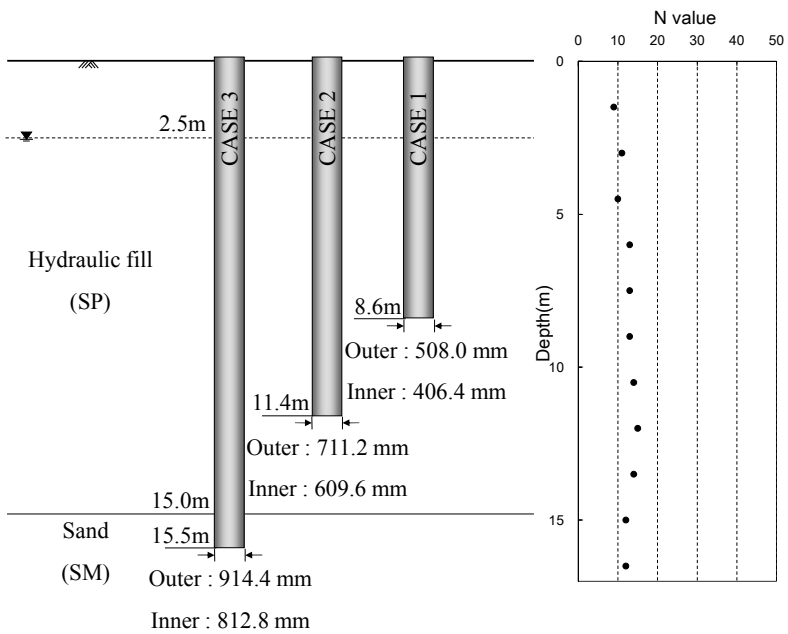

Fig. 1. Soil profile with the borehole and embedment for test piles.

Table 1. Test conditions.

\begin{tabular}{lll}
\hline Soil & Hydraulic fill & Sand \\
\hline Total unit weight, $\gamma_{t}\left(\mathrm{kN} / \mathrm{m}^{3}\right)$ & 17.6 & 18.0 \\
\hline Poisson's ratio, $\mu_{s}$ & 0.3 & 0.3 \\
\hline Friction angle, $\Phi,($ degree $)$ & 32 & 33 \\
\hline N value & $8-18$ & $12-27$ \\
\hline
\end{tabular}

\subsection{Installation of the test piles}

Full-scale field load tests were performed on three instrumented piles to investigate the relation between the plugging effect and bearing capacity.

The bearing capacity of open-ended piles consists of three components: the outer skin friction, annulus load capacity, and inner skin friction (or soil plug capacity). The instrumented double-walled pile system (Paik et al., 2003) can be applied to separate all resistance components of the open-ended piles. The instrumented piles, TP-1, TP-2, and TP-3, have outer diameters of 508.0, 711.2, and $914.4 \mathrm{~mm}$, respectively. The instrumented piles were prefabricated in factories. The gap between the outer and inner piles was welded to prevent any soil intrusion during the installation and testing. Each pile was driven by a hydraulic hammer, DKH-13 (hammer weight : $130 \mathrm{kN}$, max potential energy : $156 \mathrm{kN} \cdot \mathrm{m}$ ). To prevent potential damages of strain gauges during pile driving, the free fall height of the hammer was $0.3 \mathrm{~m}$. The final penetration depths of TP-1, TP-2 and TP-3 were recorded as 8.6, 11.4, and $15.5 \mathrm{~m}$, respectively. Automated equipment including linear variable differential transformers (LVDTs) and strain gauges were used. Two LVDTs were installed on the pile head to measure the settlement with sequential loading steps, and 32 strain gauges (electrical resistance type: 20 , vibration wire type: 12 ) per instrumented pile were installed around the pile circumference every 90 degrees to measure all components of the bearing 
capacity for the open-ended piles. These gauges were sealed with a thin membrane to prevent mechanical damage. The output from the LVDTs and strain gauges was acquired using a 200-channel carrier frequency amplifier and computer data-acquisition system.

\subsection{IFR measurement}

The degree of soil plugging in the open-ended piles affects the pile behavior. The IFR is a good indicator of the degree of soil plugging (Paik and Salgado, 2003). The IFR is defined as the increment of soil plug length per increment of pile penetration depth during pile installation. The increment of penetration depth $\left(\Delta D_{p}\right)$ was assumed as $1.0 \mathrm{~m}$. During the pile driving, the IFR was measured at each increment of $1.0 \mathrm{~m}$ penetration depth. In Fig. 2, the results from Ko and Jeong (2015) were obtained from the comparison with the IFR and N value. The inner skin friction between the soil plug and the inner piles varied, depending on the degree of soil plugging. Because of the difference in magnitude of the mobilized inner skin friction, TP-1 had a smaller IFR than the others test piles. Additionally, the IFR of all test piles had consistent trends. For all test piles, the IFR significantly decreased with the penetration depth from 0-2.5 $\mathrm{m}$ and 6-7.5 $\mathrm{m}$ and increased with the penetration depth from 2.5-6 $\mathrm{m}$ and 7.5-9 $\mathrm{m}$. This results show that the IFR increases with an increase of N, and vice versa. It is evident that the soil condition and pile diameter are highly correlated with the IFR.

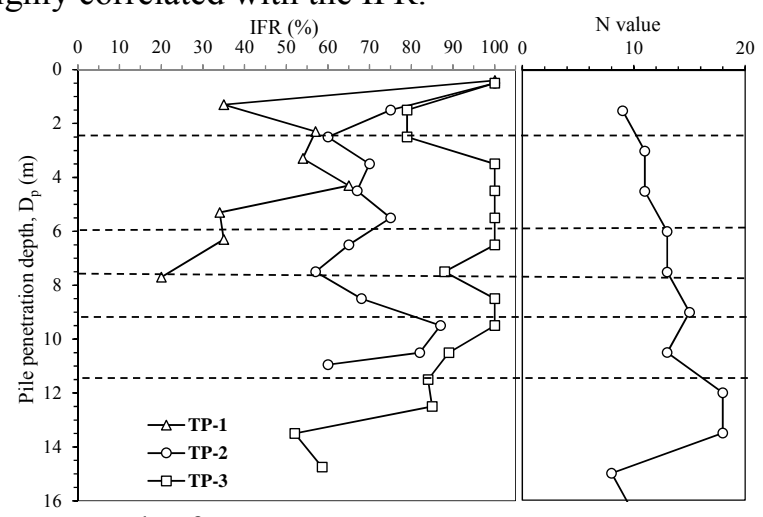

Fig. 2. Result of IFR measurements.

\subsection{Field load test}

Full-scale tests, including dynamic and static axialcompression load tests, were performed on the three instrumented piles with different diameters (508.0, 711.2 and $914.4 \mathrm{~mm}$ ). To measure the outer and inner shaft resistances that acted on the piles, a double-walled system was used with instrumented strain gauges on the outside and inside walls of the pile. Dynamic load tests were performed to evaluate the bearing capacity of the piles at the end of initial driving (EOID) following the ASTM D4945 protocol. The Case Pile Wave Analysis Program (CAPWAP) indicated that the ultimate bearing capacities of TP-1, TP-2 and TP-3 were 1,031, 2,240 and $3,100 \mathrm{kN}$ at EOID, respectively. Due to the site conditions, static axial-load tests were performed 45 days after the EOID to investigate the setup effect. The static load tests were conducted in compression based on the ASTM D1143 protocol. Twelve earth anchors were installed at the test site to serve as a reaction. The maximum loads applied were $250 \%$ of the design loads. The loading and unloading were systematically performed in 5 cycles and 10 steps. During the loading stage, each step lasted a minimum of 20 min, whereas the step duration was no less than 10 min during the unloading stage. The axial-load distribution profiles are obtained by analyzing the measured strain gauge data along the pile inside and outside. The typical test results are presented in Fig. 3 in terms of the penetration depth versus the axial-load distribution. The ultimate bearing capacities of piles TP-1, TP-2, and TP-3 were 1,000, 2,000 and 3,000 kN, respectively. As shown in Fig. 3(a), (c) and (e), it is shown that inner skin friction was mobilized within a distance of 1.3-2.3 $\mathrm{m}$ from the pile tip. It was found that the soil plug in the lower part considerably influenced on the inner skin friction. This trend is generally consistent with previous studies (Paikowsky, 1990; Feng and Jun, 2012).
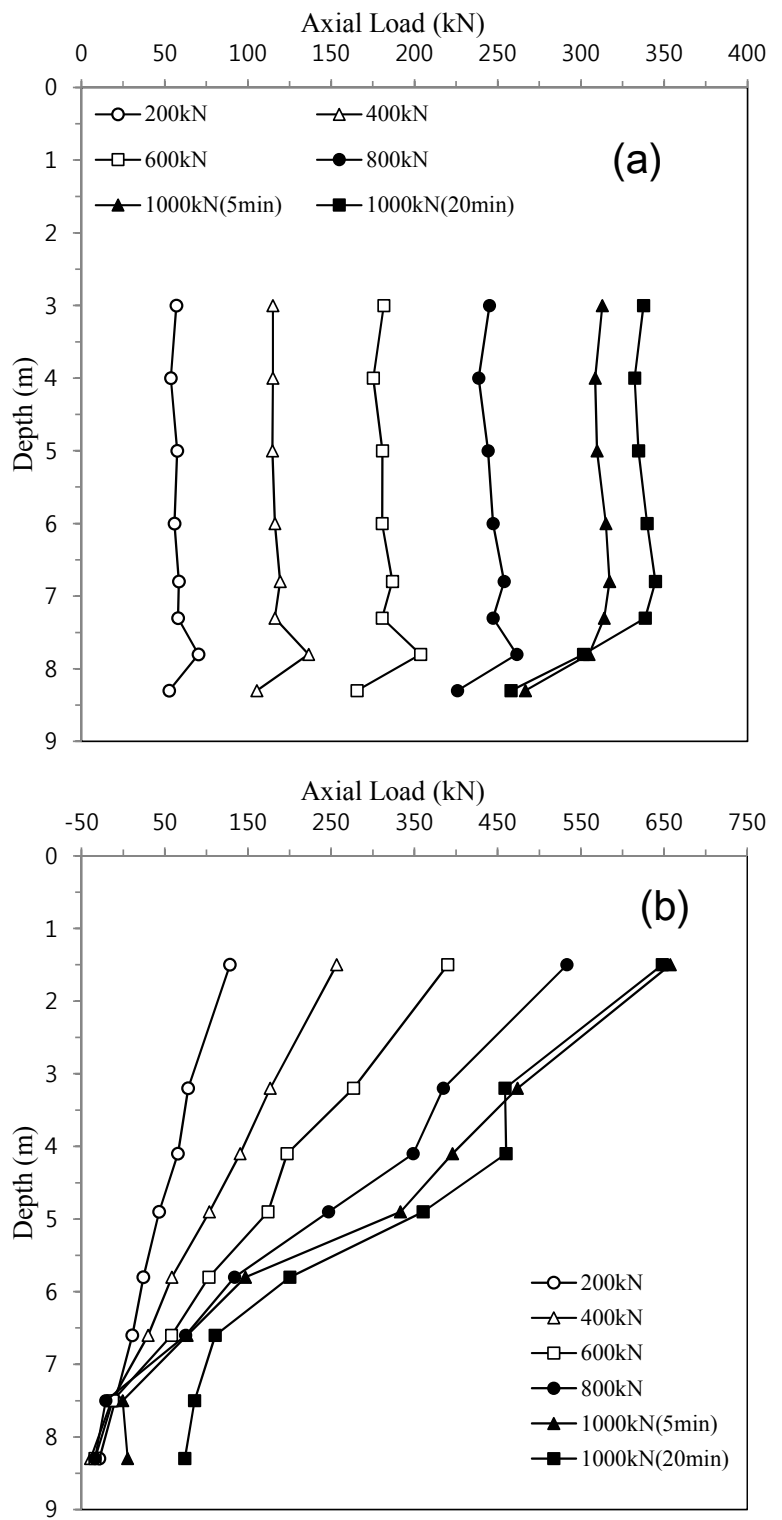


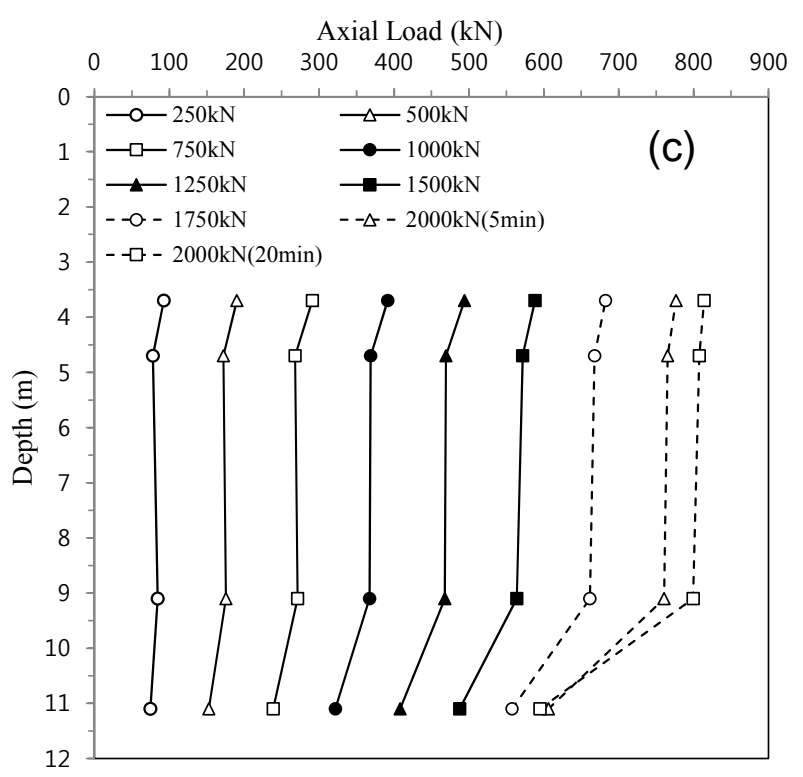

Axial Load $(\mathrm{kN})$

$\begin{array}{lllllllllllll}-200 & -100 & 0 & 100 & 200 & 300 & 400 & 500 & 600 & 700 & 800 & 900 & 1000\end{array}$

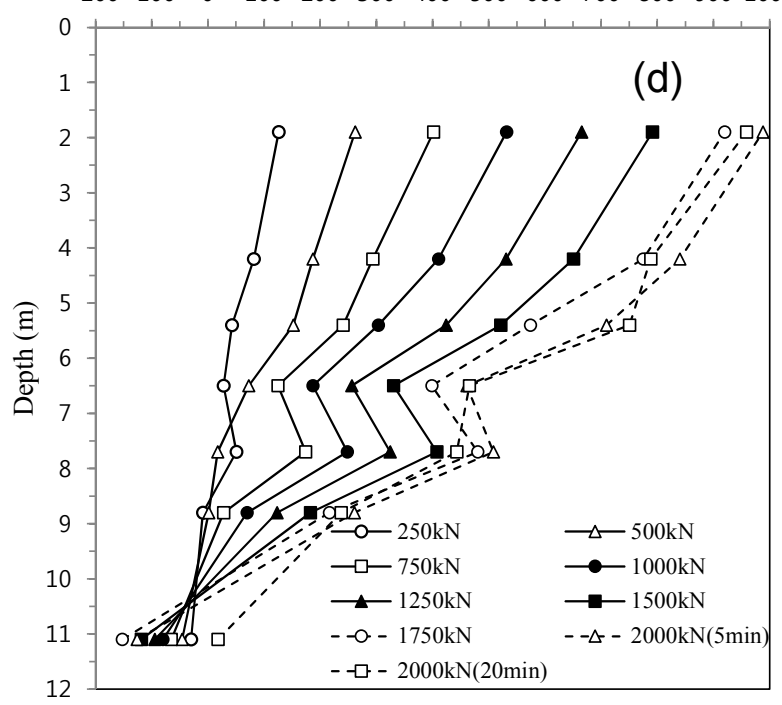

Axial Load $(\mathrm{kN})$

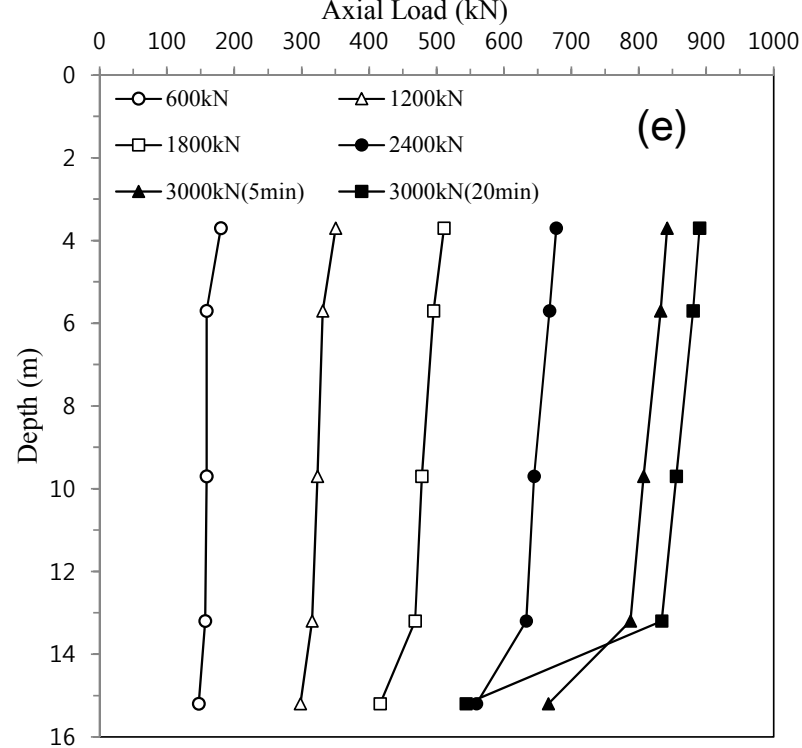

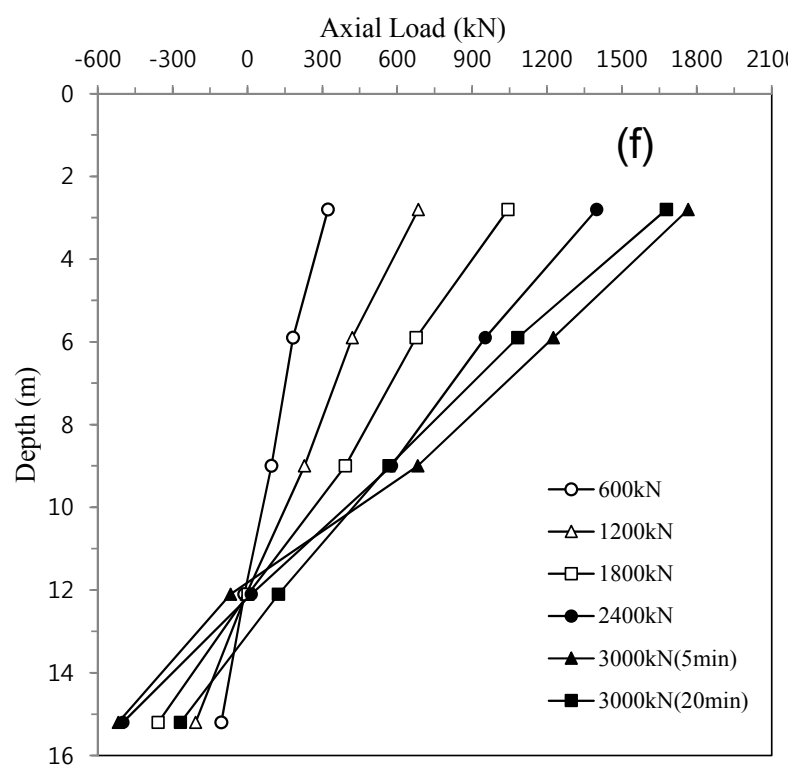

Fig. 3. Axial load distributions of inner and outer pile: (a) inner pile of TP-1; (b) outer pile of TP-1; (c) inner pile of TP-2; (d) outer pile of TP-2; (e) inner pile of TP-3; (f) outer pile of TP-3.

\section{PROPOSED RELATIONSHIP BETWEEN THE PLR AND IFR}

The PLR was measured at the end of pile driving, but the IFR was measured during the pile installation. Therefore, it is easier to obtain the PLR than the IFR in the design stage. In most previous studies, the IFR is considered proportional to the PLR (Paik et al., 2003). To investigate the relationship between the PLR and IFR, it is necessary to analyze the results of the PLR and IFR field measurements.

The PLR and IFR measurement data from three field cases were analyzed. Based on the aforementioned previous studies, a trend line for the test data can be proposed in the form of

$$
I F R=a(P L R)+b
$$

where PLR is the plugging length ratio, and $a$ and $b$ are the fitting parameters.

Fig. 4 (a) presents the relationship between the PLR and IFR based on the results obtained from the field tests reported in this manuscript. From the linear regression analysis, the parameters $\mathrm{a}$ and $\mathrm{b}$ were determined to be 110.3 and -11.5 , respectively and the following empirical equation for the relationship between the PLR and IFR can be written.

$$
I F R=110.3(P L R)-11.5
$$

In the previous studies, Paik and Salgado (2003) reported the relationship between the PLR and IFR, which can be expressed as follows for the calibration chamber tests:

$$
I F R=109(P L R)-22
$$

The fully unplugged condition can be determined by 
using PLR which is greater than or equal to 1.0 (Paik, 1994). As shown in Fig. 4 (b), two points of PLR higher than 1 were in the fully unplugged condition. Fig. 4 (b) compares this study and other studies. Equation (4) slightly overestimates the IFR for Equation (5). In addition, Equation (4) generally overestimates the IFR given by PLR values lower than 0.85 , as shown in Fig. 4 (b). It is shown that there is a difference in the trend between this study and the existing method because the pile diameter (D) of this study is larger (508.0 - 914.4 $\mathrm{mm})$ than the other field cases $(\mathrm{D}=356.0 \mathrm{~mm})$ or model cases. Therefore, the proposed relationship between the PLR and IFR given by Equation 4 was notably realistic and convenient for the preliminary design stage of openended piles.
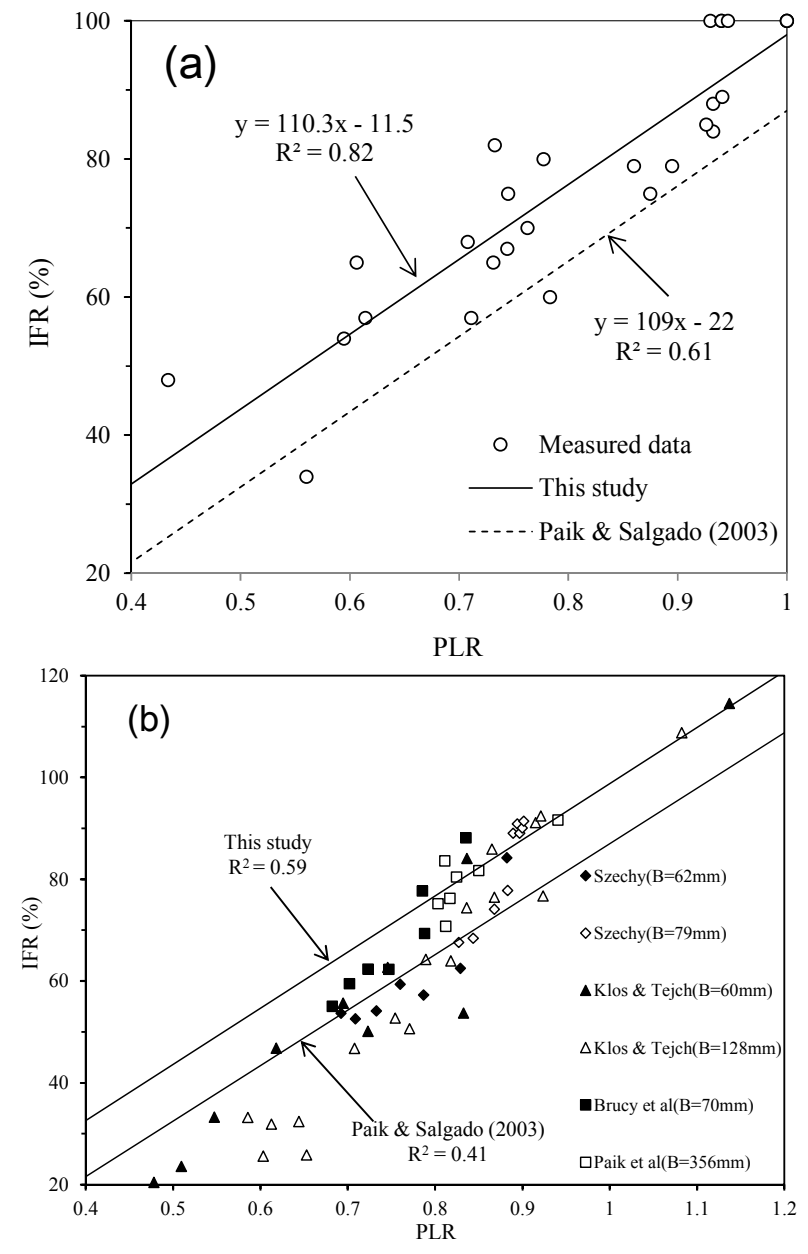

Fig. 4. The relationship between the PLR and IFR: (a) proposed IFR equation; (b) comparison with other results.

\section{PROPOSED EQUATION OF THE INNER SKIN FRICTION}

Since the 1990s, the one-dimensional plug analysis has been modified to increase in its accuracy. Randolph et al. (1991) reported the equilibrium equation for the one-dimensional plug analysis. Lehane and Gavin (2001) later reported that it was reasonable to assume that the shear stresses, which could develop at any location on the inner pile wall, were directly proportional to the vertical effective stress at that level. Because of the fast rate of excess pore water pressure dissipation, pile driven in sand is recognized as drained conditions and drained parameters are used in the analysis. The unit average skin friction can be calculated from the following equation (6) based on the analysis of effective stress.

$$
f_{s}=K_{0} \sigma_{v}{ }^{\prime} \tan \delta=\beta \sigma_{v}{ }^{\prime}
$$

where $K_{0}$ is the Rankine earth pressure coefficient, $\sigma_{v}$ ' is the effective vertical stress and $\delta$ is the angle of interface friction between the soil and inner pile wall.

This method is sensible and not overly conservative. In addition, because this method can be applied without CPT results, it is extremely useful in the preliminary design stage. However, the existing method did not consider the plugging effect.

The main objective in this research is to discover the relation between the inner skin friction and both the IFR and pile diameter. However, because there were limited data on the inner skin friction, other existing tests were used for the proposed equation of the inner skin friction. To investigate the relationship, three sets of tests are applied and analyzed. Table 2 summarizes the descriptions of the three sets.

Table 1. Test conditions.

\begin{tabular}{ll}
\hline Reference & Description \\
& Pile geometry: $\mathrm{L}=0.908 \mathrm{~m}, \mathrm{D}=42.7-89.1 \mathrm{~mm}$, \\
Paik et al. (1996) & and d=36.5-74.2 mm \\
Soil property: sand; $\mathrm{D}_{\mathrm{r}}=55$ and $90 \%$ \\
Test method: Calibrated chamber tests \\
\hline Pile geometry: $\mathrm{L}=5.2-16.7 \mathrm{~m}, \mathrm{D}=1.6 \mathrm{~m}$, and
\end{tabular}
De Nicola and $d=1.49 \mathrm{~m}$ in prototype

Randolph (1997) Soil property: silica flour; $\mathrm{D}_{\mathrm{r}}=68,85$, and $95 \%$ Test method: centrifuge chamber tests

This study Pile geometry: $\mathrm{L}=8.6-15.5 \mathrm{~m}, \mathrm{D}=0.508-0.914$ $\mathrm{m}$, and $\mathrm{d}=0.3884-0.8984 \mathrm{~m}$ in full-scale pile Soil property: sand, silty sand Test method: full-scale tests

The unit inner skin friction $f_{s i}$, which was normalized with respect to $K_{0} \sigma_{v}{ }^{\prime}$ tan $\delta$, is plotted versus (IFR- D) in Fig. 5. A trend line for the test data in Fig. 5 is proposed in the form of

$$
\frac{f_{s i}}{K_{0} \sigma_{v^{\prime}} \tan \delta}=a(I F R \times D)^{b}
$$

where $f_{s i}$ is the unit inner skin friction $(\mathrm{kPa}), K_{0}$ is the Rankine earth pressure coefficient before pile driving, $\sigma_{v}{ }^{\prime}$ is the average vertical effective stress over the entire penetration depth $(\mathrm{kPa}), \delta$ is the angle of interface friction between the soil and inner pile wall (degree), and $D$ is the pile diameter $(\mathrm{m})$. Through regression analysis, parameters a and $b$ are determined as 33.4 and -0.48 , respectively, and Equation (8) is rewritten as

$$
\frac{f_{s i}}{K_{0} \sigma_{v^{\prime}} \tan \delta}=33.4(I F R \times D)^{-0.48}
$$

The proposed equation of the inner skin friction can predict the bearing capacity considering the degree of soil plugging in the preliminary design stage. 


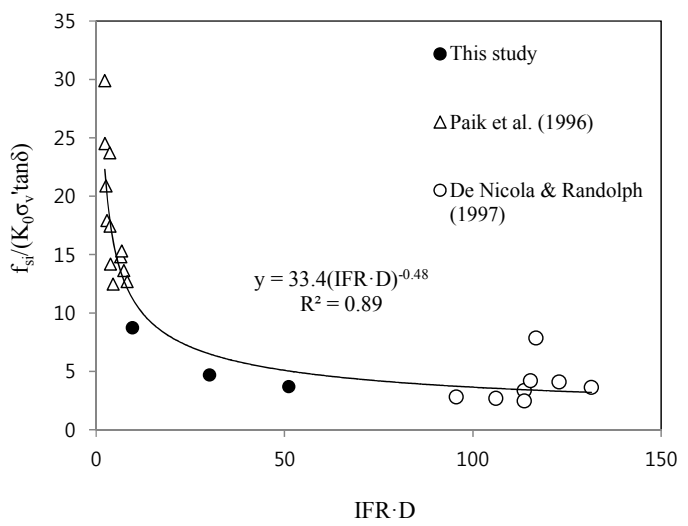

Fig. 5. Proposed equation for inner skin friction.

\section{CONCLUSIONS}

The primary objective of this study was to propose a new empirical method to quantify the bearing capacity and soil plugging (IFR) for open-ended piles. A series of field load tests and plugging measurements were conducted to investigate the plugging effect. Static axialcompression load tests were performed on three instrumented piles with different diameters (508.0, 711.2, and $914.4 \mathrm{~mm}$ ). Additionally, a regression analysis was conducted to determine the IFR, and inner skin friction equations. The following conclusions can be drawn based on the findings of this study:

1. Based on the results of the static load tests, it can be determined that most of the inner shaft resistance at the ultimate loading state was mobilised within a distance of $1.3-2.3 \mathrm{~m}$, as measured from the pile tip. The results of field tests show that the inner shaft resistance was mostly mobilized at the location between the pile tip and $18-34 \%$ of the total plug length.

2 . The proposed IFR equations can predict the degree of soil plugging in the preliminary design stage. The proposed IFR equation can be used based on the PLR when only the PLR is measured in the field.

3. The IFR is a good indicator of the degree of soil plugging. Therefore, by accounting for the IFR, the proposed inner skin friction equation is an appropriate and realistic representation of the plugging effect characteristics.

4. The proposed method, which uses the IFR and, inner skin friction, can accurately predict the degree of soil plugging of open-ended piles. The proposed design method, which consider the degree of soil plugging, can overcome the limitations of the existing design methods

\section{ACKNOWLEDGEMENTS}

This work was supported by the National Research Foundation of Korea (NRF) grant funded by the Korea government (MSIP) (No. 2011-0030040).

\section{REFERENCES}

1) API (2007): Recommended practice for planning, designing and constructing fixed offshore platforms-working stress design. 21st Ed., American Petroleum Institute, Dallas, TX.

2) ASTM (2011): Standard practice for classification of soils for engineering purpose (Unified Soil Classification System). D2487, West Conshohocken, PA.

3) ASTM (2013): Standard test methods for deep foundations under static axial compressive load. D1143, West Conshohocken, PA.

4) Brucy, F., Meunier, J. and Nauroy, J. F. (1991): Behavior of pile plug in sandy soils during and after driving. In: Proceedings of 23rd Annual Offshore Technology Conference, Houston, 1, 145-154.

5) De Nicola, A., and Randolph, M. F. (1997): The plugging behavior of driven and jacked piles in sand. Geotechnique, 47 (4), 841-856.

6) Feng Y. and Jun Y. (2012): Base Capacity of Open-Ended Steel Pipe Piles in Sand. Journal of Geotechnical and Geoenvironmental Engineering, 138 (9), 1116-1128.

7) Jardine, R. J., Chow, F. C., Overy, R. and Standing, J. (2005): ICP design methods for driven piles in sands and clays, Thomas Telford, London.

8) Klos, J., and Tejchman, A. (1981): Bearing capacity calculation for pipe piles. In: Proceedings of the 10th International Conference on Soil Mechanics and Foundation Engineering, Stockholm, Sweden, 2, 751-754.

9) Ko, J. Y., and Jeong, S. S. (2015): The plugging effect of openended piles in sandy soil [online]. Canadian Geotechnical Journal, 52, 1-13. (doi: 10.1139/cgj-2014-0041).

10) Lai, P., Mcvay, M., Bloomquist, D. and Badri, D. (2008): Axial pile capacity of large diameter cylinder piles. From Research to Practice in Geotechnical Engineering, GSP 180, ASCE, 366-384.

11) Lehane, B. M., and Gavin, K. G. (2001): Base resistance of jacked pipe piles in sand. Journal of Geotechnical and Geoenvironmental Engineering, 127 (6): 473-480.

12) Lehane, B. M., Schneider, J. A. and Xu, X. (2005): A review of design methods for offshore driven piles in siliceous sand. UWA Rep. No. GEO 05358, The University of Western Australia, Perth, Australia.

13) Paik K. H. (1994): Characteristics of bearing capacities for open-ended steel pipe piles driven into cohesionless soil. Ph.D. thesis, Korea Advanced Institute of Science and Technology, Daejeon, Korea.

14) Paik, K.H., Kim, Y.S., and Lee S.R. (1996): Effects of pile diameter on the plugging rate and bearing capacity of openended piles. Journal of the Korean Geotechnical Society, 12 (2), 85-94.

15) Paik, K.H. and Salgado, R. (2003): Determination of the bearing capacity of open-ended piles in sand, Journal of Geotechnical and Geoenvironmental Engineering, 129 (1), 4657

16) Paik, K.H., Salgado, R., Lee, J. H. and Kim, B. J. (2003): Behavior of open- and closed-ended piles driven into sands. Journal of Geotechnical and Geoenvironmental Engineering, 129 (4), 296-306.

17) Paikowsky, S. G. (1990): The mechanism of pile plugging in sand. In: Proceedings of the 22nd Offshore Technology Conference, Houston, TX, OTC 6490, 4, 593-604.

18) Randolph, M. F., Leong E. C. and Houlsby G. T. (1991): Onedimensional analysis of soil plugs in pipe piles. Geotechnique, 41 (4), 587-598.

19) Szechy, C. H. (1959): Tests with tubular piles. Acta Technica, Hungarian Academy of Science, 24, 181-219. 